\title{
Quantification of 2-butanol as a sex attractant pheromone and related alcohols emitted by individual white grub beetle, Dasylepida ishigakiensis (Coleoptera: Scarabaeidae)
}

\author{
Hiroe Yasui, ${ }^{1, *}$ Sadao WaKamura, ${ }^{1}$ Seiji Tanaka, ${ }^{1}$ Ken-ichi Harano, ${ }^{1}$ Fumiaki Mochizuki, ${ }^{2}$ \\ Atsushi Nagayama, ${ }^{3}$ Yasuhiro HoKama ${ }^{3}$ and Norio AraKaki ${ }^{3}$ \\ ${ }^{1}$ Laboratory of Insect Physiology and Behavior, National Institute of Agrobiological Sciences (NIAS); Ohwashi, Tsukuba, Ibaraki \\ 305-0851, Japan \\ ${ }^{2}$ Specialty Chemicals Research Center, Shin-Etsu Chemical Co., Ltd.; Kubiki, Joetsu, Niigata 942-8601, Japan \\ ${ }^{3}$ Okinawa Prefectural Agricultural Research Center (OPARC); Itoman, Okinawa 901-0336, Japan
}

(Received 4 June 2009; Accepted 2 September 2009)

\begin{abstract}
Females of the white grub beetle, Dasylepida ishigakiensis, emit a highly volatile, hydrophilic sex pheromone 2-butanol and related compounds. To quantify these compounds, a new method using water to trap them was developed. Vapors of 2-butanol, 2-propanol and ethanol were trapped with water, extracted with an SPME fiber and then injected into GC-MS for analyses. Quantification curves of each alcohol showed good linearity within a certain range. The recovery rate of 2-butanol released from authentic lure tubes was estimated to be ca. $70 \%$. These results indicated that the method tested would be applicable to measure the amounts of volatiles emitted by the beetles. Using this method, it was estimated that field-collected calling females emitted more than $200 \mathrm{ng}$ 2-butanol, ca. $50 \mathrm{ng}$ 2-propanol, and ca. $500 \mathrm{ng}$ ethanol on average in the laboratory. In a second calling trial, however, the amount of 2-butanol emitted by females was reduced to $3 \%$ of the amount in the first calling trial. Trace amounts of 2-butanol were detected in volatile samples from males. Because 2-propanol and ethanol were emitted by both sexes in similar amounts, they may not be involved in sexual communication.
\end{abstract}

Key words: 2-Butanol; Dasylepida ishigakiensis; hydrophilic alcohol; quantification; sex attractant pheromone

\section{INTRODUCTION}

The white grub beetle, Dasylepida ishigakiensis Niijima et Kinoshita (Coleoptera: Scarabaeidae), is one of the most destructive sugarcane pests in the Miyako Islands (Sadoyama et al., 2001). It has a semi-voltine life cycle, requiring two years to complete one generation. The larvae vigorously feed on roots and underground stems of sugarcane, which often kills the plants shortly before harvest (Oyafuso et al., 2002). The control of this insect is a prerequisite for improving sugarcane production in this area. Because this insect spends most of its life in soil, conventional control methods such as spraying may not be effective. To develop a suitable control measure for this insect, we recently determined the chemical structure of the sex pheromone (Wakamura et al., 2009a). Of the two electroantennographically (EAG) active compounds, 2-butanol and 2-propanol, which were identified from a female volatile sample, the chiral (R)-2-butanol was the only compound attractive to males in field and laboratory experiments (Wakamura et al., 2009a,b). To use this sex pheromone as a monitoring and control agent, it is important to know how much of this compound each female emits during courtship behavior. In our preliminary experiments, a conventional collection method using Tenax was found to be inapplicable, probably due to the high volatility and hydrophilicity of this compound. In an attempt to quantify various hydrophilic pollutants, including alcohols in rivers and oceans, Kadokami et al. (1996) have developed a standard method using solid phase microextrac-

* To whom correspondence should be addressed at: E-mail: yasui@affrc.go.jp DOI: 10.1303/aez.2010.129 
tion (SPME). Their studies suggested that 2-butanol and related alcohols emitted by beetles might be trapped with water before they are extracted by SPME and quantified by subsequent GC-MS analysis.

In this report, we describe a water-trapping and quantification method of highly volatile alcohols in the air, the trapping efficiency of this method, and application of this method to quantify the sex pheromone and related alcohols emitted by individual females and males. If it is known how much sex pheromone is emitted by females, effective pheromone dispensers can be developed to control this pest insect.

\section{MATERIALS AND METHODS}

Insects. Feral D. ishigakiensis beetles were collected by visual searching in sugarcane fields on Miyako Is. in the evening of February 11, 2009. Because females are usually located by males for mating soon after emergence from the soil, single females collected were considered to be virgin. Females and males were separately kept in plastic cups $(90 \mathrm{ml})$ with moist tissue paper $(<$ ca. 5 individuals/cup) and brought to the NIAS laboratory in Tsukuba. They were kept at $18^{\circ} \mathrm{C}$ under a photoperiod with light from 00:00 to 12:00 h until used.

Chemicals. 2-Butanol was purchased from Tokyo Chemical Industry Co., Ltd. (Tokyo, Japan), 2-propanol from Wako Pure Chemical Co., Ltd. (Osaka, Japan), and ethanol (99.5\%) from Nacalai Tesque Inc. (Kyoto, Japan). Potassium carbonate (Nacalai Tesque Inc.) was heated to $600^{\circ} \mathrm{C}$ for $8 \mathrm{~h}$ to release adsorbed organic chemicals. 1-Butanol$d_{9}(98 \%)$ was purchased from Cambridge Isotope Laboratories, Inc. (MA, USA). Water obtained from distillation apparatus was boiled with potassium permanganate and sulfuric acid for $24 \mathrm{~h}$ in advance, and distilled again to minimize trace amounts of 2-propanol, ethanol and other organic compounds in the original water.

Pheromone releaser (tube lure). 2-Butanol (racemic mixture) was sealed in tubes made of a copolymer of high density polyethylene and ethylene vinyl acetate $(1.30 \mathrm{~mm}$ ID and $2.34 \mathrm{~mm}$ OD). These tubes contained approximately $12 \mathrm{mg} / \mathrm{cm}$ of this compound. The tubes $0.5,1$, and $2-\mathrm{cm}$ long were kept at $20^{\circ} \mathrm{C}$ in an incubator with ventilation and were weighed every $3 \mathrm{~d}$ for $15 \mathrm{~d}$ to determine the release rate of 2-butanol from each tube $(n=3)$.

Volatile collection by glass beaker with water. (a) Collection from tube lures. Volatiles from tube lures were collected at $20^{\circ} \mathrm{C}$ in the incubator. Each glass beaker $(200 \mathrm{ml})$ was rinsed with $1 \mathrm{ml}$ distilled water and then moistened with another $1 \mathrm{ml}$ distilled water. Each 0.5, 1, and 2-cm tube lure held in a wire-mesh cage $(5 \mathrm{~cm}$ ID $\times 5 \mathrm{~cm}$ high) was introduced into a beaker which was then covered with a glass Petri dish and placed in the incubator immediately. After 10, 20, or 40 min of incubation, the lures and wire-mesh cages were removed from the beakers, and the water inside the beakers was collected individually. Each beaker was rinsed twice with ca. $1 \mathrm{ml}$ distilled water to make the final sample amount to ca. $4 \mathrm{ml}$. Water samples were sealed in $8-\mathrm{ml}$ glass vials and stored at $5^{\circ} \mathrm{C}$ until analysis $(n=6)$.

(b) Collection from D. ishigakiensis adults. Volatiles from individual $D$. ishigakiensis adults (11 females and 10 males) were collected $1 \mathrm{~h}$ before lights off. Glass beakers prepared as above were placed on a table at $22^{\circ} \mathrm{C}$. Each adult held in a wire-mesh cage was introduced into each beaker (one adult/beaker) and then covered with a glass Petri dish. The beakers were indirectly illuminated with an incandescent lamp ( $40 \mathrm{~W}$; Toshiba Co., Tokyo, Japan) which was placed above a sheet of white paper fixed $20 \mathrm{~cm}$ above the table. The initial voltage of the lamp was $130 \mathrm{~V}$, which was decreased to $100 \mathrm{~V}$ and held for $3 \mathrm{~min}$, then $80 \mathrm{~V}$, and then reduced by $10 \mathrm{~V}$ every $3 \mathrm{~min}$ until it reached $30 \mathrm{~V}$, resulting in a stepwise reduction of light intensity from 175 to $0.9 \mathrm{~lx}$, which was measured on the table using an illuminometer (TL-1; Minolta Camera Co., Ltd., Tokyo, Japan). This gradual change in light intensity was roughly equal to that encountered when mating behavior was observed in a sugarcane field (Arakaki et al., 2004; Fukaya et al., 2009). Vapor collection was conducted from 10:20 to $10: 50 \mathrm{~h}$ at $22^{\circ} \mathrm{C}$. The length of calling behavior was recorded for each female. After volatile collection, adults and wire-mesh cages were removed from the beakers, and the water samples inside the beakers were handled as above.

SPME sampling and quantification by GCMS analysis. Alcohol compounds were quantified according to the procedure described by Kadokami et al. (1996) with some modification. Water solution of 1-butanol- $d_{9}(100 \mathrm{ng} / 10 \mu \mathrm{l})$ as an internal 
standard was added to a water sample in a $8 \mathrm{ml}$ glass vial. After the addition of $4.4 \mathrm{~g}$ potassium carbonate as salting-out agent to the water sample with stirring, an SPME fiber (black: $100 \mu \mathrm{m}$ polydimethylsiloxane; Carboxen ${ }^{\mathrm{TM}}$, Supelco, Bellefonte, PA, USA) was immersed in the liquid and held for $40 \mathrm{~min}$. The SPME fiber was then lightly rinsed with water three times.

The GC-MS apparatus was a Hewlett-Packard (HP) 6890 gas chromatograph equipped with a split/splitless injector and an HP 5793 mass selective detector. A DB-WAXETR fused silica column $(50 \mathrm{~m} \times 0.32 \mathrm{~mm}$ ID $\times 1.0 \mu \mathrm{m}$ film thickness; Agilent) equipped with deactivated fused silica tubing $(1.2 \mathrm{~m} \times 0.32 \mathrm{~mm}$ ID) as a pre-column was used. Helium was used as a carrier gas at a constant flow mode of $1.1 \mathrm{ml} / \mathrm{min}$. The SPME fiber was inserted into the $\mathrm{GC}$ injection port kept at $250^{\circ} \mathrm{C}$ for $2 \mathrm{~min}$ in splitless mode and the column oven temperature was programmed from $35^{\circ} \mathrm{C}$ for $2 \mathrm{~min}$, increasing at $10^{\circ} \mathrm{C} / \mathrm{min}$ to $150^{\circ} \mathrm{C}$ for $5 \mathrm{~min}$.

The mass selective detector was operated in selected ion monitoring (SIM) mode by electron impact ionization at $70 \mathrm{eV}$. Specific ions to quantify 2butanol, 2-propanol, ethanol and 1-butanol- $d_{9}$ were measured at $\mathrm{m} / \mathrm{z} 45.0(59.0,31.0$; ions used for confirmation) at $t_{\mathrm{R}}=10.56 \mathrm{~min}, \mathrm{~m} / z \quad 45.0 \quad(59.0$, $31.0)$ at $t_{\mathrm{R}}=8.81 \mathrm{~min}, \mathrm{~m} / z 45.0(31.0)$ at $t_{\mathrm{R}}=8.99$ $\min$, and $m / z 64.1(46.1,33.1)$ at $t_{\mathrm{R}}=12.53 \mathrm{~min}$, respectively.

Calibration curves for 2-butanol, 2-propanol, and ethanol. Standard solutions of 2-butanol, 2propanol, and ethanol were prepared at $0,1,10$, 100, 500 (only 2-butanol), and 1,000 ng in $4 \mathrm{ml}$ distilled water. SPME sampling and the following GC-MS analyses were conducted using the same procedure as above. GC peak at $t_{\mathrm{R}}=8.81 \mathrm{~min}$ was 2-propanol, $t_{\mathrm{R}}=8.99 \mathrm{~min}$ ethanol, $t_{\mathrm{R}}=10.56 \mathrm{~min} 2$ butanol, and $t_{\mathrm{R}}=12.52 \mathrm{~min} 1$-butanol- $d_{9}$, respectively. Sampling and analysis at each concentration were performed more than three times. Calibration curves for each compound were calculated from area ratios of sample peaks to the internal standard peak (1-butanol- $\left.d_{9}\right)$.

Calibration curves of 2-butanol from tube lures by beaker collection and recovery rate. To evaluate the quantification method, (a) interaction between tube lengths and the amounts of 2-butanol collected from the lures, and (b) interaction between collection periods and the amounts of 2-bu- tanol collected from the lures were investigated. In (a), volatile collection from $0.5-, 1-$, and 2-cm lures was conducted for $20 \mathrm{~min}$. In (b), volatiles from a $1-\mathrm{cm}$ lure were collected for $0,10,20$, and $40 \mathrm{~min}$. SPME sampling and subsequent GC-MS analyses were conducted as above. Sampling and analysis for each condition was performed six times. The amount of collected 2-butanol was calculated from the area ratios of sample peaks to the internal standard peak (1-butanol- $d_{9}$ ). Based on these results, calibration curves were drawn for the amounts of collected 2-butanol relative to lure lengths or collection periods. The recovery rate of 2-butanol collected from lures by the glass-beaker collection method was determined based on weight changes in the tube lure at $20^{\circ} \mathrm{C}$.

Statistics. The amount of collected compounds from beetles was analyzed using $t$-test (Fig. 3). The amounts of female emitted compounds collected every two days were submitted to one-way-layout analysis of variance. The means were ranked by Tukey's method when ANOVA was significant at $p=0.05$. Values accompanied by the same letter within the same compound are not significantly different at $p=0.05$ (Fig. 5).

\section{RESULTS}

Collection of authentic 2-butanol, 2-propanol, and ethanol from water solution

Various amounts of authentic 2-butanol, 2propanol, and ethanol were added to water separately, and each alcohol was collected by SPME and analyzed by GC-MS (SIM). Calibration curves for 2-butanol, 2-propanol, and ethanol are shown in Fig. 1. Good linearity was obtained for 2-butanol within a range from 1 to $100 \mathrm{ng}(\log Y=1.15 \cdot \log X$ $-0.15, r^{2}=0.991$, range $1-100 \mathrm{ng}$ ), where $X$ is the amount (ng) of the compound, and $Y$ is the percentage of the peak area of the compound to that of $100 \mathrm{ng}$ internal standard (1-butanol- $\left.d_{9}\right)$, but the measured values deviated downward beyond this range. Therefore, another calibration curve was drawn within a range from 100 to $1,000 \mathrm{ng}$ that showed good linearity $(\log Y=0.78 \cdot \log X+$ $0.55, r^{2}=0.983$, range $100-1,000 \mathrm{ng}$ ). Because 2propanol and ethanol were detected in untreated distilled water, calibration curves for the compounds did not go through the origin. Calibration curves were calculated as follows; 2-propanol: 

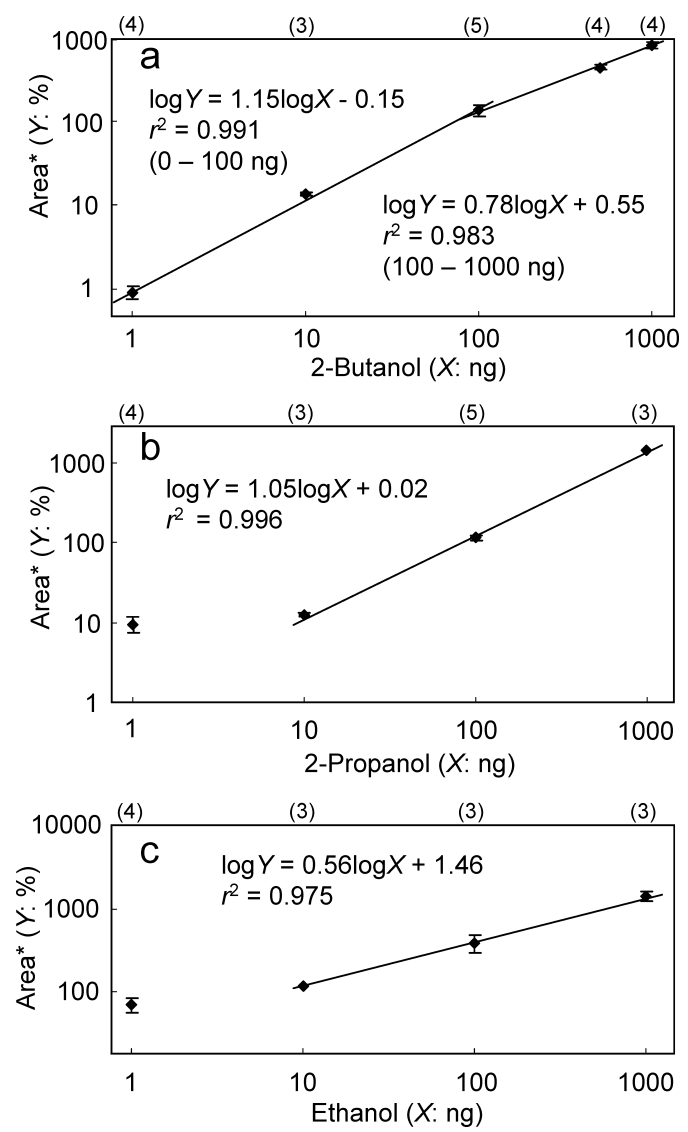

Fig. 1. Overall recovery of authentic alcohols, 2-butanol, 2-propanol, and ethanol from water solution by SPME. a: 2butanol, b: 2-propanol, c: ethanol. Bar: SD. Numbers in parentheses indicate sample sizes. * Percentages of peak area of $\mathrm{m} / \mathrm{z}$ $45.0 / \mathrm{m} / z$ 64.1 (1-butanol- $d_{9}$ ).

$\log Y=1.05 \cdot \log X+0.02 \quad\left(r^{2}=0.996, \quad\right.$ range $\quad 10$ $1,000 \mathrm{ng}$ ), and ethanol: $\log Y=0.56 \cdot \log X+1.46$ $\left(r^{2}=0.975\right.$, range $\left.10-1,000 \mathrm{ng}\right)$.

\section{Overall recovery rate of 2-butanol by the water- trapping method}

To evaluate the efficacy of the water-trapping method, 2-butanol released from tube lures of different lengths for various time periods was extracted with water in beakers. The amount of collected 2-butanol was directly related to the lure lengths (Fig. 2a, $Y=507 X+271, r^{2}=0.995$ ) and the length of time spent for extraction (Fig. 2b, $Y=$ $33 X, r^{2}=0.988$ ). The mean amounts of 2-butanol extracted from $0.5-, 1-$, and $2-\mathrm{cm}$ tube lures by the water-trapping method were $26 \mathrm{ng} / \mathrm{min}, 38 \mathrm{ng} / \mathrm{min}$, and $64 \mathrm{ng} / \mathrm{min}$, respectively. To determine the release rate of 2-butanol from the tube lures, the
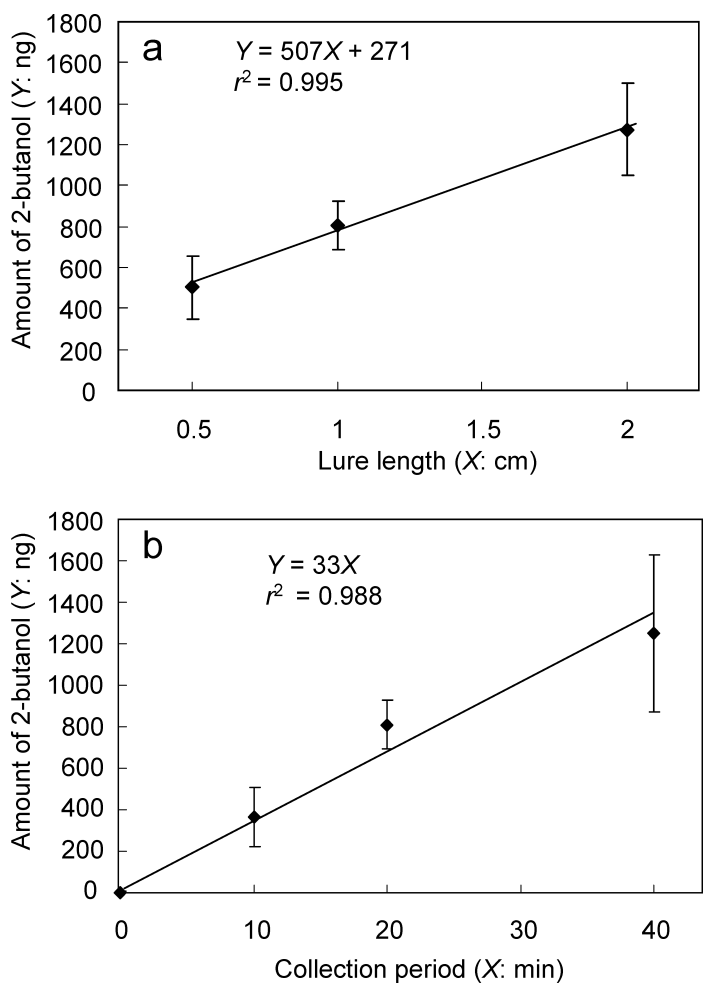

Fig. 2. Quantification of 2-butanol from tube lures using the water-trapping method. a: Collection from three different lengths of lures $(0.5,1,2 \mathrm{~cm})$ for $20 \min (n=6)$, b: collection from $1 \mathrm{~cm}$ lure for three different periods $(10,20,40 \mathrm{~min}$, $n=6)$. Bar: SD.

same types of tube lures were kept at $20^{\circ} \mathrm{C}$ and their weight changes were monitored for $15 \mathrm{~d}$. The average weight decreases of $0.5-, 1-$, and 2-cm tube lures were $30 \pm 8 \mathrm{ng} / \mathrm{min}, 49 \pm 4 \mathrm{ng} / \mathrm{min}$, and $81 \pm 10$ $\mathrm{ng} / \mathrm{min}$, respectively (mean $\pm \mathrm{SD}, n=3$, each). These results indicated that the recovery rate of 2butanol by the water-trapping method was ca. $70 \%$.

\section{Quantification of alcohols emitted by adult $D$. ishigakiensis}

Eleven field-collected females individually confined to wire-net cages were held in glass beakers to collect volatile samples with water on $17 \mathrm{Feb}$. 2009, the first calling after being collected in the field on 11 Feb. 2009. When illumination was gradually reduced to ca. $50 \mathrm{~lx}$, they started moving their abdomen (typical calling behavior, Arakaki et al., 2004; Fukaya et al., 2009) on the wire nets. 2Butanol was detected from all volatile samples from females with the mean amount being $210 \pm$ $140 \mathrm{ng}$ (mean $\pm \mathrm{SD}, n=11$; Fig. 3). 2-Propanol 


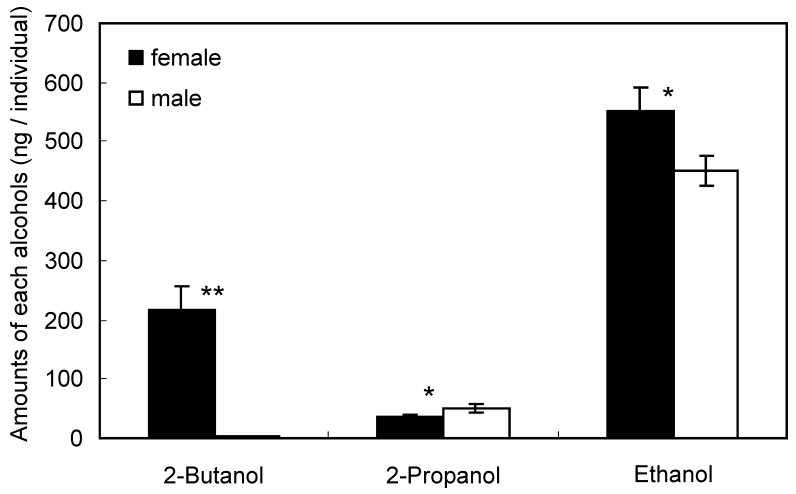

Fig. 3. Amount of alcohols emitted by adult D. ishigakiensis. Mean amounts of each compound were analyzed using $t$-test. ${ }^{*} p<0.05,{ }^{*} p<0.01$. Bar: SE. Sample sizes are 11 and 10 in females and males, respectively.

(35 $\pm 12 \mathrm{ng})$ and considerable amounts of ethanol $(550 \pm 130 \mathrm{ng})$ were also detected in volatile samples.

Volatiles from ten males were also collected individually as for females on 18 Feb., and trace amounts of 2-butanol were detected $(1.8 \pm 0.8 \mathrm{ng})$. The mean amount was more than a hundred times smaller than that from females, and the difference was statistically significant at $p<0.001$ by $t$ test (Fig. 3). 2-Propanol $(50 \pm 25 \mathrm{ng})$ and ethanol $(450 \pm 78 \mathrm{ng}$ ) were also found in the male volatiles, as observed in female samples and a significant sex difference was found in either compound at $p<$ 0.05 .

\section{Relationship between calling period and amount of pheromone emitted by females}

The relationship between calling periods and the amounts of emitted 2-butanol was analyzed using the same data obtained from female volatiles collected on 17 Feb. The mean calling period was 12.6 \pm 4.0 min (mean $\pm \mathrm{SD}, n=11$, Fig. 4). All females assumed a calling posture and the calling period ranged from 3 to $18 \mathrm{~min}$. The amount of collected 2-butanol was above $100 \mathrm{ng}$ with a mean value of $210 \mathrm{ng}$ (SD, $140 \mathrm{ng}$, Fig. 4). No significant correlation was found between the amounts of 2butanol collected and the lengths of calling behavior $\left(r^{2}=0.043, p>0.50\right.$, Fig. 4$)$.

\section{Daily emission of 2-butanol by feral females}

To determine how much pheromone can be emitted by single females after being collected in the field, ten female beetles collected on 16 Feb.

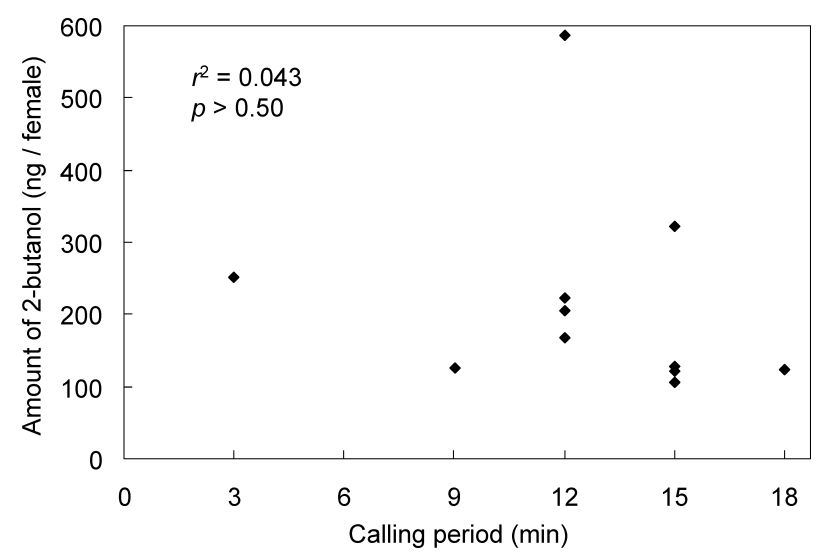

Fig. 4. Relationship between calling period and amount of 2-butanol emitted by D. ishigakiensis females. $n=11$.

2009 were divided into two groups and the amount of 2-butanol emitted from each female was determined. In one group (group A, $n=5$ ), 2-butanol was collected and quantified every other day from 17 Feb. (day 1) until 25 Feb. (day 9). In the other group (group $\mathrm{B}, n=5$ ), pheromones were sampled on days 1,3 , and 7 . In group $\mathrm{A}$, the mean amount of 2-butanol collected was $210 \pm 140 \mathrm{ng}$ (mean \pm SD) on day 1 , but markedly decreased to $9.2 \pm 6.3$ ng on day 3 (Fig. 5A). The subsequent changes were rather small; $8.4 \pm 3.5 \mathrm{ng}$ on day $5,5.6 \pm 1.5 \mathrm{ng}$ on day 7 , and $6.3 \pm 2.3 \mathrm{ng}$ on day 9 . In group $\mathrm{B}$, the same tendency was observed: the amount of 2-butanol collected was $282 \pm 67 \mathrm{ng}$ on day 1 and $10.6 \pm$ $3.2 \mathrm{ng}$ on day 3 . The last sampling 4 days later (day 7) amounted to $19.7 \pm 0.9 \mathrm{ng}$ but this value was not significantly different from that on day 3 in the same group.

\section{DISCUSSION}

To quantify the sex pheromone emitted by the female white grub beetle $D$. ishigakiensis, a trapping method was developed for the highly volatile compound. The present study revealed that female $D$. ishigakiensis emits not only the sex pheromone, 2-butanol and an EAG active component 2propanol (Wakamura et al., 2009a), but also a considerable amount of ethanol. An initial attempt to detect these alcohols in samples extracted by organic solvent was unsuccessful because the GC peaks of those compounds overlapped with the solvent peak. Therefore, solvent extracts of females could not be used for chemical identification of the 

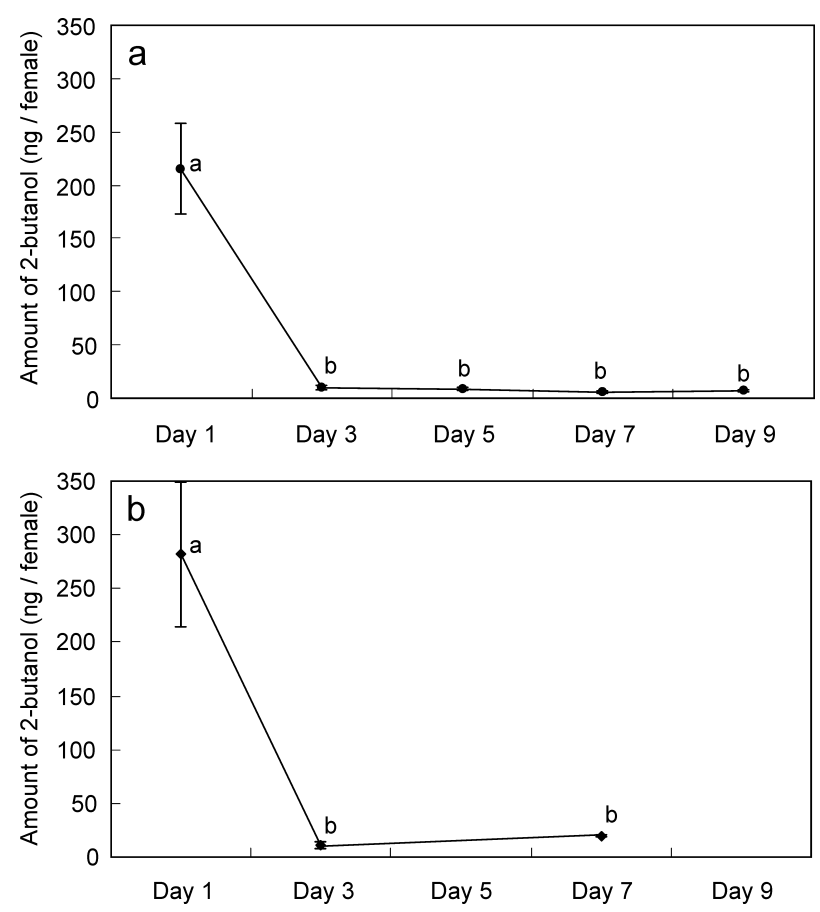

Fig. 5. Daily changes in amounts of 2-butanol emitted by $D$. ishigakiensis females after being collected in the field. a: on every 2 days $(n=5)$, b: on Days 1,3 , and $7(n=5)$. Values accompanied by the same letter are not significantly different at $p=0.05$ level by one-way-layout ANOVA and subsequent Tukey's ranking. Bar: SE.

pheromone candidate for this species. The present study modified the reported method for determining hydrophilic alcohols in water samples (Kadokami et al., 1996) and used a small volume of water for collecting the volatiles emitted by this beetle; that is, volatiles emitted by female beetles were trapped with water in a glass beaker, the water sample with an internal standard was salted out with potassium carbonate and an SPME fiber was inserted into the sample to adsorb the alcohols. The SPME fiber was then injected into GC-MS to quantify the alcohols.

In a previous study (Wakamura et al., 2009a), 2butanol was detected from aerial sampling using a SPME fiber into a beaker in which $D$. ishigakiensis females were calling, and the amount of 2-butanol collected was estimated to range from $<20 \mathrm{pg}$ to ca. $3 \mathrm{ng}$. However, this estimation was rather inaccurate, because it was not known how efficiently the SPME fiber trapped or extracted the volatiles in the beaker. In the present study, the volatiles were first trapped in water and the water samples were analyzed. As a result, a linear relationship was found between the amounts of added target alcohols and ratios of SIM peak area of the alcohols to that of the internal standard (1-butanol- $\left.d_{9}\right)$. The result suggests that reliable quantitative analysis of these compounds is possible by SPME (Fig. 1).

To evaluate the efficiency of the water-trap method, 2-butanol released from tube lures in beakers was extracted with water and the amount of compound in the water samples was analyzed in relation to the length of tube lures and duration of the releasing period. Good linearity was obtained between the amounts of collected 2-butanol and tube lengths or release periods (Fig. 2). This and other results indicated that the recovery rate of 2butanol by this method was approximately $70 \%$.

2-Propanol is another alcohol emitted by females but it does not attract male adults; however, it shows a dose-dependent inhibitory effect on male attraction when blended with $(R)$-2-butanol in a field test (Wakamura et al., 2009a,b). Ethanol was also found in female volatiles in a relatively large amount, but it was not attractive to males (unpublished data). Because 2-propanol and ethanol are also found in male volatile samples (Fig. 3), they may not be involved in sexual communication. Although a trace amount of 2-butanol was detected in male volatiles, the amount is vanishingly small compared to the significant amount of the same compound emitted by females.

No significant relationship was observed between calling periods and the amounts of emitted pheromone by females (Fig. 4), i.e., females that assumed a calling posture for longer did not always emit larger amounts of pheromone. One possibility is that although females assume a calling posture for a long time, they might emit the pheromone only within a limited time, e.g., during the early phase of calling behavior. According to field observations, males can find a female as soon as she emerges from the soil and perches on vegetation (Arakaki et al., 2004). This, together with the fact that the sex ratio of field populations is biased toward males (unpublished data), suggests that females might not need to keep emitting the pheromone for a long time.

In the black chafer, Holotrichia loochooana loochooana (Coleoptera: Scarabaeidae), large individual and daily fluctuations have been observed in the release amount of the pheromone compound 
(0-400 ng/individual, Yasui et al., 2007). On the other hand, in D. ishigakiensis, individual variation in the amount of the pheromone compound was not so large (within an order of magnitude) but the amount decreased markedly after the first calling in the laboratory. In a second calling trial, females emitted only $3 \%$ of the amount at the first calling trial. Because the females used in this study were collected on Miyako Is. at the beginning of the emerging season, they were likely to have come out for the first calling meaning that the first calling in the laboratory in Tsukuba was probably their second calling. Arakaki et al. (2004) reported that mated females return to the soil and seldom come out again for a second mating. This may suggest that females of this beetle do not call more than once if they mate successfully on the first calling night. However, field-collected virgin females which called at least once in the field emitted significant amounts of the sex pheromone in the laboratory, although we do not know how much pheromone females should emit for successful mating. A marked reduction in the release amount at the second calling onward in the laboratory is probably due to the exhaustion of beetles, which consume no food as adults.

\section{ACKNOWLEDGMENTS}

We thank Hiroaki Oroku and Sumisu Sakiyama (present address, Nago Branch of OPARC) of the Miyakojima Branch of OPARC, for their kind support of this project.

\section{REFERENCES}

Arakaki, N., Y. Sadoyama, M. Kishita, A. Nagayama, A. Oyafuso, M. Ishimine, M. Ota, T. Akino, M. Fukaya, Y. Hirai, K. Yamamura and S. Wakamura (2004) Mating behavior of the scarab beetles Dasylepida ishigakiensis (Coleoptera: Scarabaeidae). Appl. Entomol. Zool. 39: 669-674.

Fukaya, M., H. Yasui, T. Akino, T. Yasuda, S. Tanaka, S.
Wakamura, T. Maeda, Y. Hirai, K. Yasuda, A. Nagayama and N. Arakaki (2009) Environmental and pheromonal control of precopulatory behavior for synchronized mating in the white grub beetle, Dasylepida ishigakiensis (Coleoptera: Scarabaeidae). Appl. Entomol. Zool. 44: 223-229.

Kadokami, K., K. Sato, T. Iwamura and Y. Hanada (1996) Determination of hydrophilic alcohols from aquatic environment by solid-phase microextraction and GC/MS. Bunseki Kagaku 45: 1013-1018 (in Japanese with English summary).

Oyafuso, A., N. Arakaki, Y. Sadoyama, M. Kishita, F. Kawamura, M. Ishimine, M. Kinjo and Y. Hirai (2002) Life history of the white grub Dasylepida sp. (Coleoptera: Scarabaeidae), a new and severe pest on sugarcane on the Miyako Islands, Okinawa. Appl. Entomol. Zool. 37: 595-601.

Sadoyama Y., H. Oroku and H. Nakamori (2001) Injury of sugar cane roots by the white grub, Dasylepida sp. (Coleoptera: Scarabaeidae), in the Miyako Islands. Jpn. J. Appl. Entomol. Zool. 45: 89-91 (in Japanese with English summary).

Wakamura, S., H. Yasui, T. Akino, T. Yasuda, M. Fukaya, S. Tanaka, T. Maeda, N. Arakaki, A. Nagayama, Y. Sadoyama, M. Kishita, A. Oyafuso, Y. Hokama, A. Kobayashi, K. Tarora, T. Uesato, A. Miyagi and J. Osuga (2009a) Identification of ( $R$ )-2-butanol as a sex attractant pheromone of the white grub beetle, Dasylepida ishigakiensis (Coleoptera: Scarabaeidae), a serious sugarcane pest in the Miyako Islands of Japan. Appl. Entomol. Zool. 44: 231-239.

Wakamura, S., H. Yasui, F. Mochizuki, T. Fukumoto, N. Arakaki, A. Nagayama, T. Uesato, A. Miyagi, H. Oroku, S. Tanaka, M. Tokuda, M. Fukaya, T. Akino, Y. Hirai and M. Shiga (2009b) Formulation of highly volatile pheromone of the white grub beetle Dasylepida ishigakiensis (Coleoptera: Scarabaeidae) for development of monitoring trap. Appl. Entomol. Zool. 44: 579-586.

Yasui, H., S. Wakamura, N. Arakaki, T. Yasuda, T. Akino and M. Fukaya (2007) Collection and quantification of airborne pheromone from individual females of the black chafer Holotrichia loochooana loochooana (Coleoptera: Scarabaeidae): Heterogeneity of feral females in respect to pheromone release. Appl. Entomol. Zool. 42: 143150 . 